\title{
Bent to Bind: Exploiting the Programmed Cell Death-1 (PD-1) Recep- tor Plasticity to Design Pembrolizumab H3 Loop Mimics
}

\author{
Mehdi Zaghouani ${ }^{a}$, Guangkuan Zhao ${ }^{a}$, Alexis D. Richaud ${ }^{a}$, Medhi Wangpaichitr ${ }^{c}$, Niramol Savaraj ${ }^{c}$, \\ and Stéphane P. Roche $\mathrm{e}^{a, b *}$ \\ ${ }^{a}$ Department of Chemistry and Biochemistry, Florida Atlantic University, Boca Raton, Florida 33431, USA \\ ${ }^{b}$ Center for Molecular Biology and Biotechnology, Florida Atlantic University, Jupiter, Florida 33458, USA \\ ${ }^{c}$ University of Miami School of Medicine, Miami, Florida 33458, USA
}

KEYWORDS: Programmed Death-1 (PD-1); Pembrolizumab mimicry; Protein plasticity; Conformational motion; Antibody hypervariable loops; Drug binding; PD-1 Inhibitors; $\beta$-hairpin peptide inhibitor.

\begin{abstract}
Checkpoint blockade of the Programmed cell Death-1 (PD-1) immunoreceptor with its ligand 1 (PD-L1) by the monoclonal antibody pembrolizumab provided compelling clinical results among various cancer types, yet the molecular mechanism by which this drug blocks the PD-1:PD-L1 binding interface and reactivates exhausted T cells remains unclear. To address this question, we examined the conformational motion of PD-1 associated with the binding of pembrolizumab. The largely overlooked innate plasticity of both PD-1 C'D and FG loops appears crucial to closing in the receptor edges on the drug. Herein, we describe how PD-1 bends to initiate the formation of a deep binding groove $\left(371 \AA^{3}\right)$ across several epitopes while engaging pembrolizumab. Our analysis ultimately provided a rational design for mimicking the pembrolizumab H3 loop [RDYRFDMGFD] as a PD-1 inhibitor. A series of $\mathrm{H} 3$ loop mimics were synthesized and their folding characterized by $\mathrm{CD}$ and NMR spectroscopy. As a result, a first-in-class $\beta$-hairpin peptide inhibitor of the PD-1/PD-L1 interface was identified $\left(\mathrm{IC}_{50}\right.$ of $0.6 \pm 0.2 \mu \mathrm{M})$. Overall, this study demonstrates that the dynamic groove formed between the C'D and FG loops of PD-1 is an attrac-
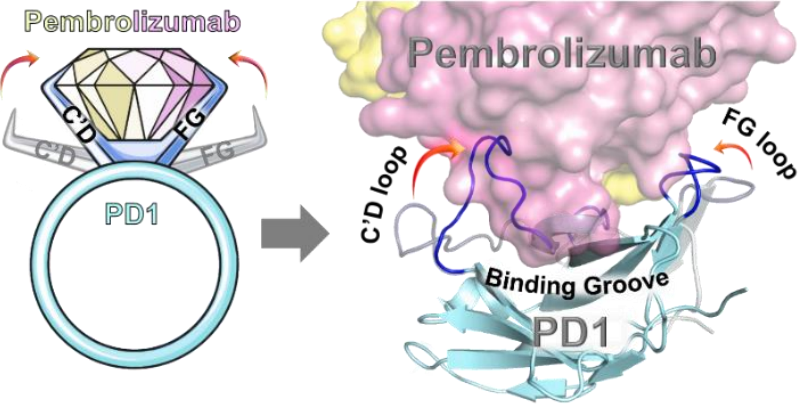
tive target for the development of peptide-based PD-1 inhibitors.
\end{abstract}

Checkpoint inhibitors have recently drawn considerable interest in cancer immunotherapy as modulators of the immune response. ${ }^{1,2}$ Tumors have been shown to target certain immunecheckpoint mechanisms to evade detection by activated $\mathrm{T}$ cells. The PD-1 receptor (also known as CD279) associated with one of its natural ligand PD-L1 (i.e. CD274 or B7-H1) was established as a major checkpoint exploited by cancer cells to suppress the immune response. ${ }^{3-5}$ Indeed, cancer tissues inhibit the host immune response by upregulating PD-L1 which in turn leads to the exhaustion of antigen-specific $\mathrm{CD}^{+} \mathrm{T}$ cells in the tumor microenvironment. Monoclonal antibodies (mAbs) blocking the PD-1:PD-L1 checkpoint have been shown to restore T-cell function and rapidly emerged at the forefront of one of the most significant breakthrough in cancer (immuno)therapy. ${ }^{6,7}$ Pembrolizumab, the second FDA-approved PD-1 blocker ${ }^{8}$ is being currently tested in various cancer types and over 750 active clinical trials. ${ }^{9}$ Despite this success, only a fraction of patients show a durable and complete response to these agents ${ }^{10-12}$ and some immune-related adverse events have been reported, ${ }^{13,14}$ thus suggesting that effective and safer small-molecule inhibitors of the PD-1 pathway are needed. Our current understanding of the pembrolizumab affinity to PD-1 is also limited, underlying the need to seize molecular insights into the PD- 1 activity modulation. ${ }^{15}$ Therefore, we became interested in generating a comprehensive structural comparison between all available high-resolution crystal structures of PD-1 in complex with pembrolizumab and PD-L1 to investigate the differences in interactions at the molecular level (residues, patches/segments, and surface). ${ }^{16}$ Based on this new structural analysis, we were able to set a clear definition of 3 epitopes on PD-1 targeted

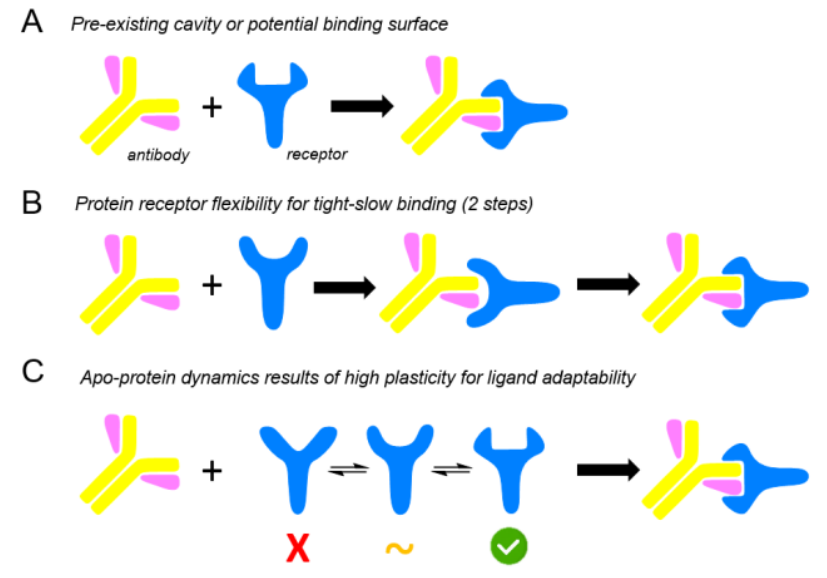

Figure 1. Distinct mechanisms for protein-ligand binding: (A) lock-andkey model. (B) Koshland's induced-fit model. (C) conformational selection followed by induced-fit model, as proposed herein for the PD1:pembrolizumab binding.

by pembrolizumab and the dynamic nature of their binding interface(s) (Figure 1). Our study also revealed important insights into the nature of conformational dynamics responsible for forming a groove between the C'D and FG loops of PD-1 upon binding to pembrolizumab. These results enabled the reinterpretation of the PD-1:pembrolizumab interactions recently proposed by Wang and Song, ${ }^{17}$ and suggested that the (complementary determining region heavy-chain) CDR-H3 loop could inform the design of peptide-based PD-1 inhibitors. While large $\mathrm{mAb}$ blockers have demonstrated impressive clinical results, only a handful of small-molecule inhibitors of PD-L1 have been 
developed, and no PD-1 inhibitors have been reported to date. ${ }^{18-}$ ${ }^{22}$ Using a technology for the synthesis of $\beta$-hairpins displaying long loops recently developed by our group ${ }^{23}$, several mediumsize molecules mimicking the H3-loop of pembrolizumab $\left[{ }^{99}\right.$ RDYRFDMGFD $\left.{ }^{108}\right]$ were synthesized and evaluated as PD1 inhibitors in vitro. As a result, the first-in-class peptide $\beta$-hairpin inhibitor $1 \mathrm{c}$ reported herein $\left(\mathrm{IC}_{50}\right.$ of $0.6 \mu \mathrm{M}$ ) validated a new promising modality for the design of PD-1 inhibitors.

\section{Results and Discussion}

Role of PD-1 C'D and FG Plasticity in Forming a Binding Groove at the Interface with Pembrolizumab. The conformational plasticity of proteins has emerged as a compelling strategy to enable the recognition of multiple ligands at a single molecular surface (e.g. mostly same set of surface residues and patches) to modulate biological functions. The importance of protein plasticity is increasingly recognized to play a major role in protein-ligand binding, conformational selection and in epitope mapping ${ }^{24-27}$ in comparison to static models, yet the plasticity of PD-1 has been largely overlooked until now. Given that binding of PD-1 to PD-L1 was shown to be entropicallydriven by ligand approach, ${ }^{28,29}$ we thought to closely examine the potential binding modes of pembrolizumab to PD-1, such as lock-and-key, induced-fit, and dynamic conformational-fit mechanisms (Figure 1A-C). ${ }^{30,31}$ Interestingly, a molecular dynamics (MD) simulation study by Liu and coworkers suggested that the flexibility of the CC' loop in apo-PD-1 is enhancing a conformational pre-equilibrium of the receptor prior to binding PD-L1, similar to a conformational selection followed by induced-fit mechanism (Figure 1C).$^{32}$ Although, the considerably higher affinity of PD-1 for PD-L2 rather than PD-L1 remained unexplained for years, the recent study by Kim and Tang revealed that binding to PD-L2 triggered a major conformational motion of the PD-1 CC' and FG loops to create a favorable binding pocket at the surface of PD- $1 .{ }^{33}$ Along these lines, the recent study by Goa and coworkers on anti-PD-1 mAbs also suggested that the PD-1 FG loop plasticity played a crucial role in the binding event of blockers and provided a novel targetable region. ${ }^{34}$ Taken together, these results suggested that apo-PD-1 present a substantial innate plasticity in the unbound state suitable to conformational selection, leading to our hypothesis that binding to pembrolizumab could operate through a dynamic conformational-fit mechanisms (Figure 1C).

The lack of complete structural and conformational information on the pembrolizumab:PD-1 interface has hampered the rational design of PD-1 inhibitors. At first, epitopes on the PD1 surface were mapped to decipher the entire binding interface with pembrolizumab (Figure 2). Since the initial interpretation by Wang and Song of the "hot spot" residues essential to the protein-protein interaction (PPI) between the PD-1 ectodomain and the antigen-binding fragment (Fab) of pembrolizumab (PDB code: 5JXE, $2.9 \AA$ resolution),${ }^{17}$ the acquisition of higher resolution crystal structures by Nomura (PDB code: 5B8C, 2.1 $\AA$ resolution) ${ }^{35}$ and most recently by Heo (PDB code: 5GGS, $2.0 \AA$ resolution $)^{36}$ enabled a more accurate description of the protein-ligand interface. Yet, several assumptions in the interpretation of the initial low-resolution crystal structure -in which water molecules and the conformationally flexible ${ }_{\text {PD-1 }} C^{\prime}$ 'D loop backbone were unresolved- thwarted the initial investigation of the main antigen-binding sites (paratopes) of pembrolizumab responsible for the drug affinity to PD-1. From our analysis, the PD-1:pembrolizumab interface could be divided into 3 main sub-surfaces (Figure 2, see also Supplementary Table S4 and
Figure S1). ${ }^{37}$ Epitope-1 formed by residues of the ${ }_{\mathrm{PD}-1} \mathrm{CC}$ ' $\beta$ hairpin (Asn ${ }^{66}, \mathrm{Tyr}^{68}, \mathrm{Gln}^{75}$ (only in 5B8C) $\mathrm{Thr}^{76}, \mathrm{Asp}^{77}$ and $\mathrm{Lys}^{78}$ ) was found to interact with several complementary determining regions (CDRs) of pembrolizumab, notably with residues from the CDR-H1 $\left(\mathrm{Thr}^{\mathrm{H} 30}, \mathrm{Tyr}^{\mathrm{H} 33}\right)$, CDR-H2 $\left(\mathrm{Asn}^{\mathrm{H} 52}\right.$, $\mathrm{Ser}^{\mathrm{H} 54}$ ) and CDR-H3 (Tyr $\left.{ }^{\mathrm{H} 101}, \mathrm{Arg}^{\mathrm{H} 102}\right)$. This highly localized epitope also contains a large hydrophobic core which corresponds to the hinge region of the PD-1 curvature. Interestingly, earlier computational ${ }^{32}$, and NMR binding studies ${ }^{29}$ as well as crystallographic structures of the PD-1:PD-L1 complex ${ }^{38}$ (PDB code: $4 Z \mathrm{ZK}, 2.4 \AA$ resolution $)^{39}$ already suggested that the $\mathrm{PD}$ ${ }_{1} \mathrm{CC}$ ' $\beta$-hairpin of epitope- 1 was essential to the native ligand PD-L1 recognition (See also Supplementary Video S1). As a competitive inhibitor of PD-L1, the higher affinity of pembrolizumab $\left(K_{d}=29 \mathrm{pM}\right)^{40}$ could not be attributed solely to the interactions generated at epitope- 1 as initially suggested..$^{17}$ In fact PPI blockers must typically exhibit large surfaces of interactions for competitive binding with the native ligand, ${ }^{41}$ therefore a closer analysis of paratopes- 2 and -3 was deemed necessary.

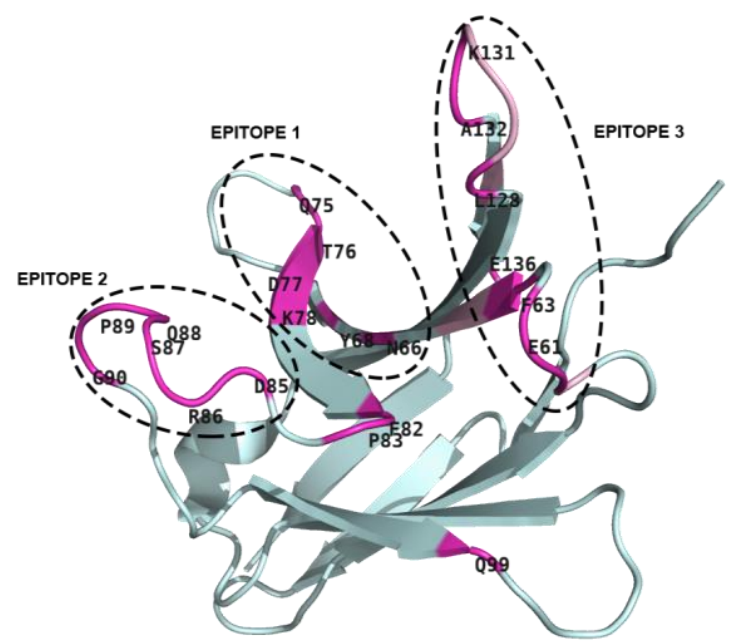

Figure 2. Mapping and partitioning of PD-1 epitopes at the binding interface with pembrolizumab (structure of pembrolizumab-fab omitted for clarity). Ribbon representation of PD-1 excised from the co-crystal 5B8C with patches of residues involved in contacts shown in pink.

Epitope-2 was mapped entirely to the ${ }_{\mathrm{PD}-1} \mathrm{C}^{\prime} \mathrm{D}$ loop $\left[\mathrm{Phe}^{82}-\right.$ $\mathrm{Phe}^{95}$, which displayed interactions with the CDRs H1-3 and CDR-L1,3 (Figure 2). Because the C'D loop does not engage in any binding interactions with PD-L1 (PDB: 4ZQK), its coiled structure is highly flexible and disordered, similar to the C'D loop in apo-PD-1 (PDB: 3RRQ at $2.1 \AA$ resolution). In stark contrast, in all the reported crystal structures of pembrolizumab:PD-1 complexes, the C'D loop is extremely structured and in extended interactions with the CDR-H3 of pembrolizumab. In order to analyze the conformational changes induced upon binding with pembrolizumab, we remodeled the C'D loop of apo-PD-1 using Chimera to obtain the lowest-energy state (from 500 low-energy conformers, Supplementary Figure S4). We then engaged this de Novo PD-1 structure bearing the energy-minimized C'D loop (PDB file $3 \mathrm{RRQ}^{* *}$ ) in $\mathrm{MD}$ and morphing simulations to assess the dynamic nature of the various loops. ${ }^{42,43}$ The morphing simulation generated by Pymol clearly established the role of the ${ }_{\mathrm{PD}-1} \mathrm{C}^{\prime} \mathrm{D}$ loop motion when binding to pembrolizumab (see Supplementary Video S2). While in the original report, $\mathrm{Asp}^{85}$ was shown to create a salt bridge with $\mathrm{Arg}^{\mathrm{H} 99}$, and residues $\mathrm{Ser}^{87}$ and Gly ${ }^{90}$ hydrogen bonded to $\mathrm{Arg}^{\mathrm{H} 99}$ and $\mathrm{Thr}^{\mathrm{H} 58}$, 4-6 new contacts interactions were 
later reported from higher resolution crystals (5B8C and 5GGS). For instance, residue $\mathrm{Glu}^{84}$ was shown to create contacts with $\mathrm{Tyr}^{\mathrm{L} 36}$ and $\mathrm{Ser}^{\mathrm{L} 95}$ (only in 5GGS), the salt bridge was consolidated with an external hydrogen bond to $\mathrm{Asp}^{\mathrm{H} 104}$, with close proximity contacts $\operatorname{Arg}^{86}: \mathrm{Asn}^{\mathrm{H} 59}, \mathrm{Gln}^{88}: \mathrm{Asp}^{\mathrm{H} 52, \mathrm{H} 55, \mathrm{H} 59}$, $\mathrm{Pro}^{89}: \mathrm{Asp}^{\mathrm{H} 52, \mathrm{H} 55}$, and $\mathrm{Gln}^{91}$ with $\mathrm{Thr}^{\mathrm{H} 58}$ and $\mathrm{Asn}^{\mathrm{H} 59}$ (only in 5GGS). Those hydrogen bonds mainly involved the backbone amides of the C'D loop residues with the CDR-H2 of pembrolizumab through water molecules. Due to the low-resolution crystal diffraction of the pembrolizumab:PD-1 complex (5JXE), water molecule coordinates could not be determined accurately, leaving several "hot" spots from solvent-exposed interactions between the ${ }_{\mathrm{PD}-1} \mathrm{C}$ 'D loop and pembrolizumab unexplained (sub-interface I in reference (17); epitope-2 in this work). Our morphing simulation further suggested that the binding of pembrolizumab required a pivotal folding of the highly hydrated ${ }_{\mathrm{PD}-1} \mathrm{C}^{\prime} \mathrm{D}$ loop surface. The flexible nature of the C'D loop was further confirmed by a $500 \mathrm{~ns}$ molecular dynamics (MD) simulation of the reconstituted apo-PD-1 and the bounded PD-1 structures using the ToeLoop algorithm (See Supplementary Figure S6). ${ }^{44,45}$ According to the calculated MD trajectories, the C'D loop mobility was characterized by "fast" dynamics. The overall flexibility of this 14 residues loop [Phe ${ }^{82}$ $\left.-\mathrm{Phe}^{95}\right]$, as well as the distance between segment termini $\left(\mathrm{d}_{\mathrm{Phe82}}\right.$ Phe95 $=11.7 \AA$ ) in comparison to the longest $\mathrm{C} \alpha$ - $\mathrm{C} \alpha$ distance within the loop $\left(\mathrm{d}_{\text {Pro83-Arg94 }}=16.3 \AA\right)$ are characteristic features of a $\Omega$-type loop. ${ }^{37,46,47}$ We showed that the conformational transition from the open-form of apo-PD-1 to the closed-form of the receptor complexing pembrolizumab (Figure 3) was characterized by a closure of $12.1 \AA$ between the C'D and FG loops ( $\mathrm{C} \alpha$ atoms of $\mathrm{Pro}^{89}$ and $\mathrm{Lys}^{131}$ ). The formation of a binding groove between the CC', C'D and FG loops of PD-1 fits the approach of the CDR-H3 loop of pembrolizumab. Collectively, these results suggest that the distal ${ }_{\mathrm{PD}-1} \mathrm{C}^{\prime} \mathrm{D}$ loop (epitope-2) and PD-1 FG loop (epitope-3) are the main structural elements affording the necessary plasticity to PD-1 for the receptor folding onto pembrolizumab (Figure 3, See also Supplementary Video S2).

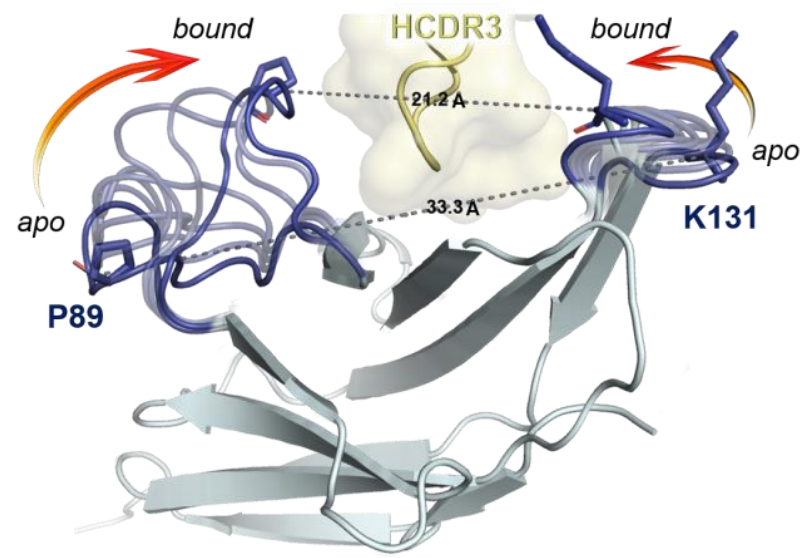

Figure 3. Conformational motion of the PD-1 C'D and FG loops upon binding to pembrolizumab. Overlay of 7 selected conformers extracted from the conformational motion simulation forming the binding groove around the pembrolizumab H3 loop (yellow). The central region of PD-1 is shown in grey with the loops motion in light blue and both extreme open and closed conformations in dark blue. Pro ${ }^{89}$ and Lys ${ }^{131}$ on each edge of the receptor are represented to show the $\mathrm{C} \alpha-\mathrm{C} \alpha$ distance closing the groove onto pembrolizumab. see Supplementary Video S2

Epitope-3 is composed of patches from the ${ }_{\mathrm{PD}-1} \mathrm{BC}$ and ${ }_{\mathrm{PD}-1} \mathrm{FG}$ loops and residue $\mathrm{Val}^{64}$ from the $\mathrm{C}$ strand which generated a large surface of contacts with the corresponding CDR-H3, CDR-L1 and CDR-L2 regions of pembrolizumab. Overall, hydrogen-bond contacts from $\mathrm{Leu}^{128}: \mathrm{Tyr}^{\mathrm{H} 53}, \mathrm{Ala}^{129}: \mathrm{Leu}^{\mathrm{H} 58}$ and a salt bridge Lys $^{131}: \mathrm{Glu}^{\mathrm{L} 59}$ (CDR-L2), and 3 other hydrogen bonds from $\mathrm{Lys}^{131}$ and $\mathrm{Ala}^{132}$ to $\mathrm{Met}^{\mathrm{H} 105}, \mathrm{Asp}^{\mathrm{H} 108}$ and $\mathrm{Arg}^{\mathrm{H} 102}$, and $\mathrm{Val}^{64}: \mathrm{Phe}^{\mathrm{H} 103}$ (CDR-H3) were observed. These data are consistent with the important role of the ${ }_{\mathrm{PD}-1} \mathrm{FG}$ loop previously demonstrated by mutagenesis studies of PD- $1,{ }^{48}$ and therefore highlights the significance of the pembrolizumab CDR-L2. Interestingly, we found that paratope-3 is highly lipophilic $(\sim 10$ van der Walls contacts) due to a segment of the CDR-L 2 rich in leucine and tyrosine residues $\left(\mathrm{Tyr}^{\mathrm{L} 53}, \mathrm{Leu}^{\mathrm{L} 54}, \mathrm{Tyr}^{\mathrm{L} 57}\right.$, and $\mathrm{Leu}^{\mathrm{L} 58}$ ) in addition to $\mathrm{Gly}^{33}$ and $\mathrm{Tyr}^{34}$ from the CDR-L1.

Collectively, the morphing and MD simulations demonstrated that the two distal epitopes 2-3 (C'D and FG loops) display an important elasticity across the receptor (Supplementary Videos S2-S3). The conformational analysis also revealed that the CDR-H2 makes a number of contacts with the folded $\Omega$ type C'D loop of PD-1, thus engaging fully with the PD-1 target through a solvent-exposed surface. The entropic penalty associated with the folding of PD-1 to form a binding groove at the interface with pembrolizumab is likely offset by the enthalpic contributions from a large number of polar contacts and the two salt bridges created on each side of the pembrolizumab CDRH3 with epitope-2 (Asp ${ }^{85-}: \mathrm{Arg}^{\mathrm{H99+}}{ }^{-}$and CDR-L2 with epitope-3 $\left(\mathrm{Lys}^{131+}: \mathrm{Glu}^{\mathrm{L} 59-}\right.$ ).

Rational Design of the Pembrolizumab $\mathrm{H3}$ loop mimics. Our comprehensive structural and conformational analysis of the pembrolizumab:PD-1 interface revealed that the CDR-H3 hypervariable loop (H3 loop) of pembrolizumab interacts with all three epitopes of PD-1, further suggesting that the [ $\mathrm{Arg}^{\mathrm{H} 99}$ $\mathrm{Asp}^{\mathrm{H} 108}$ ] patch is likely the most important binding pharmacophore (see binding surfaces in Figure S1). The full-length pembrolizumab antibody was originally reported by Merck scientists to be unusually compact and rigid (PDB: 5DK3 at $2.3 \AA$ resolution), thus imparting a high degree of preorganization to the entire Fab Fv-region in solution. ${ }^{8}$ As shown in Figure 4A, the rigidity and compactness of the $\mathrm{H} 3$-loop backbone was established by aligning the unbound pembrolizumab antibody (5DK3, in blue) with the 3 pembrolizumab-Fab excised from PD-1 X-ray co-crystal structures. Next, we calculated the conformational root mean square deviations (RMSD) of the superimposed loops to the unbounded loop over residues $\mathrm{Arg}^{\mathrm{H} 99}$ to $\mathrm{Asp}^{\mathrm{H} 108}$. The loop motif is well defined as shown by the mean RMSD values of $0.22 \pm 0.06 \AA$ for the 10 loop $\mathrm{C} \alpha$ atoms, and of $0.35 \pm 0.13 \AA$ over the entire 40 backbone atoms. In addition to the number of internal hydrogen bonds present across the loop, these RMSD values suggest that the H3 loop is highly rigid, compact and conformationally constrained.

This [Arg $\left.{ }^{\mathrm{H} 99}-\mathrm{Asp}^{\mathrm{H} 108}\right] \mathrm{H} 3$-loop motif offers a suitable spatial distribution of binding "hot" spots, thereby enabling interactions with the three epitopes across the PD-1 receptor (Figure 4). Due to the low-resolution of the 5JXE crystal structure, and the lack of defined water molecules in this complex, fewer contacts can be observed (Figure 4B), but our interpretations of the 5B8C and 5GGS high-resolution crystals afforded more information. For instance, the H3 loop engaged not only with a groove in epitope-1 $\left(\mathrm{Tyr}^{\mathrm{H1} 101}: \mathrm{Thr}^{76}, \mathrm{Arg}^{\mathrm{H1} 102}: \mathrm{Asn}^{66}\right.$, and $\mathrm{Tyr}^{\mathrm{H} 101} / \mathrm{Tyr}^{\mathrm{H} 102}: \mathrm{Tyr}^{68} / \mathrm{Lys}^{78}$ ), but also with residues in epitope-2 $\mathrm{Arg}^{\mathrm{H} 99}: \mathrm{Ser}^{87}$, the salt bridge $\mathrm{Arg}^{\mathrm{H} 99} / \mathrm{Asp}^{\mathrm{H} 104}: \mathrm{Asp}^{85}$, and with epitope-3 residues $\mathrm{Tyr}^{\mathrm{H} 101} / \mathrm{Arg}^{\mathrm{H} 102}: \mathrm{Glu}^{136}, \mathrm{Arg}^{\mathrm{H} 102}: \mathrm{Ala}^{132}$, 
$\mathrm{Phe}^{\mathrm{H} 103}: \mathrm{Val}^{64}$, and $\mathrm{Met}^{\mathrm{H} 105} / \mathrm{Asp}^{\mathrm{H} 108}: \mathrm{Lys}^{131}$ (Figure 4C-D). Furthermore, the total buried surface area (BSA) at the pembrolizumab/PD-1 interface was estimated to $1,218 \AA^{2} .{ }^{37}$ From this interface, we found that a large surface of interactions $\left(715 \AA^{2}\right)$ was created by the antibody heavy-chain region. Strikingly, our data suggest that the binding of pembrolizumab to PD-1 is heavily associated to the CDR-H3 domain, as the $\left[\mathrm{Arg}^{\mathrm{H} 99}-\mathrm{Asp}^{\mathrm{H} 108}\right]$ loop surface itself accounts for $38 \%$ of the overall BSA (460 $\AA^{2}$ ). The dimensions of the groove formed between the C'D and FG loops of PD-1 were calculated by POCASA 1.1 using a probe sphere of $6 \AA$ radius adapted for shallow cavities. ${ }^{49}$ The entire groove volume was estimated to $371 \AA^{3}$ (Supplementary Figure S2). Our calculations support an earlier hypothesis from Iwata and Nomura who postulated that the 16-residue $\beta$-hairpin peptide $\left[\mathrm{Cys}^{\mathrm{H} 96}-\mathrm{Gly}^{\mathrm{H} 111}\right.$ ] might be essential to the pembrolizumab binding mechanism. ${ }^{35}$ Taken together, the rigidity of the $\mathrm{H} 3$ loop [ $\mathrm{Arg}^{\mathrm{H} 99}-\mathrm{Asp}^{\mathrm{H} 108}$ ] and the number of contact interactions with residues pertaining to all three epitopes of PD-1 over an extended BSA justified the design of pembrolizumab CDRH3 mimics as novel scaffolds for developing PD-1 inhibitors.
Chemical Synthesis, Characterization and Biological Evaluation of $\boldsymbol{\beta}$-Hairpin Inhibitors of PD-1. A number of "hot" loops found at antibody-protein interfaces,,${ }^{50,51}$ are potentially very attractive targets for developing novel drug blockers. Yet the design of inhibitors of dynamic PPIs amenable to initiating conformational selection by induced-fit adjustment, like the PD-1:PD-L1 checkpoint interface described herein remains challenging. ${ }^{52}$ Our comprehensive binding analysis suggested the CDR-H3 peptide fragment $99-108$ of pembrolizumab as a suitable binder of PD-1 (Figure 4C-D). Therefore, a synthesis of $\beta$-hairpin mimics was undertaken by exploiting a technology of stabilizing $\beta$-straps developed in our laboratory. ${ }^{23} \beta$-Hairpins displaying long loops $(\mathrm{N} \geq 10$-mer) are inherently challenging to prepare due to the large entropic penalty associated with their loop nucleation and folding. ${ }^{53,54}$ For this reason, we recently developed several stabilizing $\beta$-strap motifs specifically crafted for long loops to create amphipathic structures with hydrophobic residues such as W/W pairs on one face and ionic residues on the other.
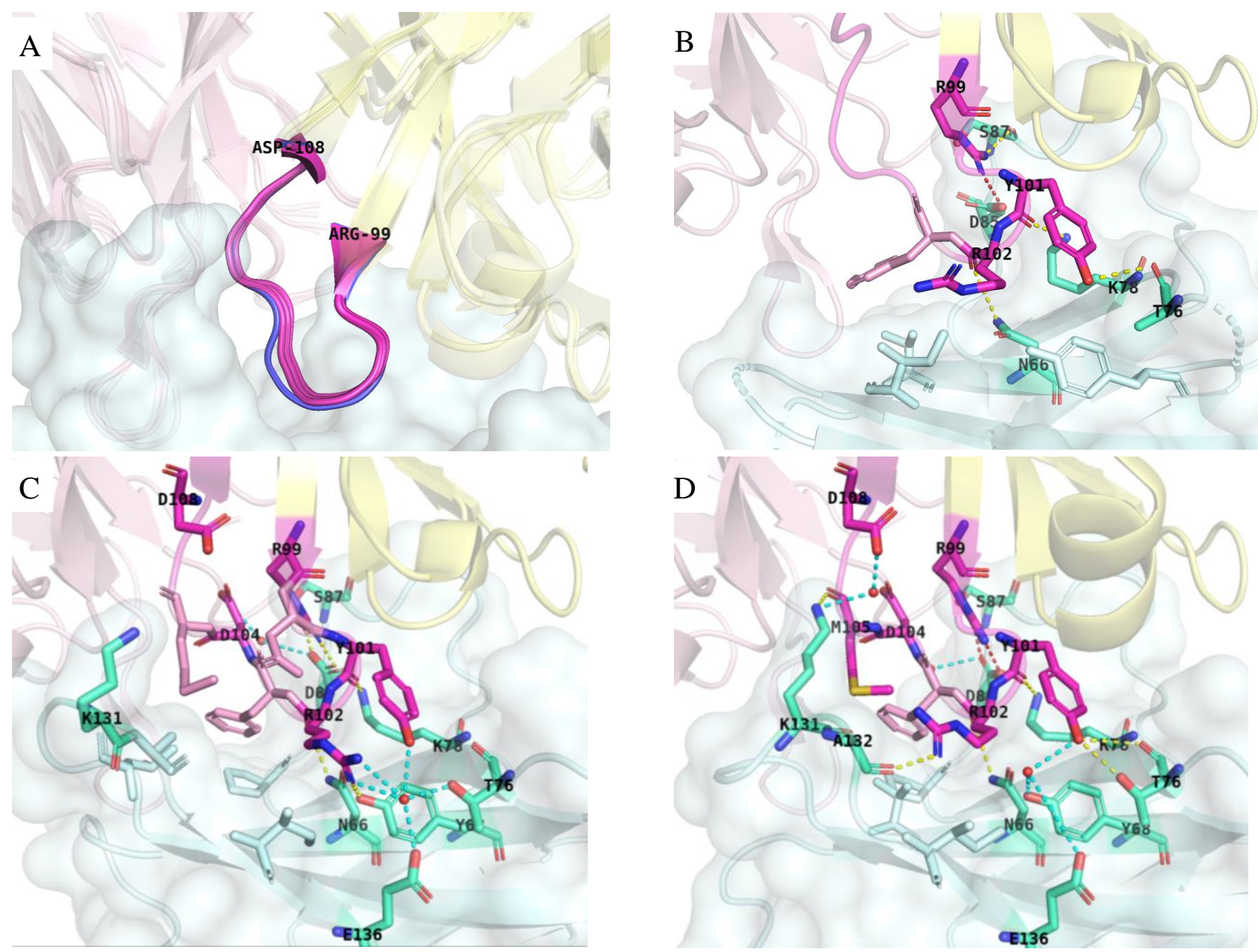

Figure 4. Comparison of binding modes from close-up views of pembrolizumab CDR-H3 loop at the interface with PD-1. (A) Overlay of pembrolizumab $\mathrm{H} 3$ loops $\left[\mathrm{Arg}^{\mathrm{H} 99}-\mathrm{Asp}^{\mathrm{H} 108}\right.$ ] excised from co-crystal structures (PDB: 5JXE, 5B8C, 5GGS, in magenta) with the unbounded form (PDB: 5DK3, in blue), corresponding RMSD values of $0.28 \AA, 0.15 \AA, 0.22 \AA$ over $\mathrm{C} \alpha$ atoms, and $0.48 \AA, 0.26 \AA, 0.30 \AA$ over backbone atoms respectively calculated against the unbounded structure. (B-D) Ribbon representation of binding complexes from PDBs 5JXE, 5B8C and 5GGS are shown with PD-1 in cyan, and with the heavy and light chains of pembrolizumab in yellow and pink respectively. The network of contact interactions created by the $\mathrm{H} 3$ loop in each complex is depicted with H-bonds between H3 loop and PD-1 residues in magenta and green cyan respectively, and hydrophobic contacts in the same lighter colors. Direct and water mediated H-bonds are displayed with yellow and cyan dashed lines and salt bridges with red dashed lines. 
To display the 10-residue loop, we evaluated the two most stabilizing $\beta$-strap RW(VW) $\bullet \cdot(\mathrm{WV}) \mathrm{WE}$ motifs constituted of a hydrogen bond network, a hydrophobic core and a terminal salt bridge. As a control, the native hairpin sequence excised from pembrolizumab $R W$-CAR-[RDYRFDMGFD]-YWG-WE 1a was prepared with the shortest strap. Two other analogs such as $\mathbf{1 b}$ in which the $C^{3} / V^{18}$ pair was replaced by a V/V pair, and 1c bearing a longer strap motif were also prepared to determine the stabilizing effect of the strap either placed after a short $\beta$-strand or directly flanking the loop. By inserting two extra glycine residues in the loop a more flexible analog $\mathbf{1 d}$ was built around the second $\beta$-strap motif Ac-W $\bullet \bullet W T G-\mathrm{NH}_{2} \cdot{ }^{53}$ Finally, we synthesized two analogs 1e,f carrying simple unfunctional polyglycine $\mathrm{G}_{4} \mathrm{KKG}_{(2 / 4)}$ loops which will serve as negative controls to ascertain relationships between biological activity, loop sequences and the conformational topology of these hairpins.

We then determined the structure and folding features of these constructs 1a-f, by a combination of far UV-CD (circular dichroism) and NMR (nuclear magnetic resonance) spectroscopy (Figure 5). Due to Trp/Trp facing residues inside the $\beta$ straps (EtF indole rings), a characteristic and intense exciton couplet comprised of a negative and a positive maxima at 212 and $228 \pm 1 \mathrm{~nm}$ should be observed on the CD spectra. ${ }^{55} \mathrm{As}$ shown in Figure 5A, the original peptide sequence 1a directly excised from pembrolizumab presented no such folding exciton. In addition, peptide 1a was found to be poorly soluble in aqueous solutions even with the addition of organic cosolvents (DMSO or MeOH). Similarly, the CD spectra of peptides 1d and $\mathbf{1 f}$ harboring flexible loops showed no band at $228 \pm 1 \mathrm{~nm}$, but a distinctive band at $200 \pm 1 \mathrm{~nm}$ indicative of random coil conformations, thus establishing that no hairpin fold was obtained in solution (Supplementary Figure S8). In contrast, the intense exciton at $228 \pm 1 \mathrm{~nm}$ from the highly flexible construct 1e supported the formation of a hairpin secondary structure (Figure 5A). This result suggested that the $\mathrm{RW}(\mathrm{VW}) \cdots(\mathrm{WV}) \mathrm{WE} \beta$-strap played an important stabilizing role. Excitingly, both constructs 1b-c also exhibited several spectral features expected for $\beta$-hairpins with a characteristic band at $213 \pm 1 \mathrm{~nm}$ typical of $\beta$-sheet, and the exciton couplet at $212 / 228 \pm 1 \mathrm{~nm}$ from the $\operatorname{Trp} / \operatorname{Trp} \beta$-strap interaction. Peptide concentrations in phosphate buffer $(\mathrm{pH}$ 6.5) were accurately measured by UV absorbance to achieve a reliable quantification of hairpin folding as a function of temperature by variable-temperature CD spectral analysis (Figure 5B). The decrease in amplitude of the $228 \mathrm{~nm}$ exciton upon heating was exploited to measure the hairpin unfolding transition inside the $\beta$-strap. The changes in intensity of the molar ellipticity $[\theta(\mathrm{T})]_{228}$ indicated a temperature-induced unfolding of 1e from a typical hairpin/coil transition in agreement with a two-state model (folded/unfolded). Having generated the curves of $[\theta(T)]_{228}$ for peptides 1b-f, the plots of \%-folding as a function of temperature were obtained for each hairpin (see Supplementary Figure S9), along with their melting temperatures $T_{m}$ and the thermodynamic parameters characterizing the unfolding transition $\left(\Delta G_{\mathrm{F}}{ }^{\circ}, \Delta H_{\mathrm{F}}{ }^{\circ}\right.$, and $\left.\mathrm{T} \Delta S_{\mathrm{F}}{ }^{\circ}\right) .{ }^{56}$ Collectively, results from our VT-CD study demonstrate that constructs $\mathbf{1 b}, \mathbf{c}$ and $\mathbf{1 e}$ are significantly wellfolded hairpins at ambient temperature given their respective $T_{m}$ of $36.9,23.8$, and $51.8 \pm 0.1{ }^{\circ} \mathrm{C}$ and thermal stability curves (Figure 5C). ${ }^{37}$ Whereas hairpin 1e with a flexible loop was found to be well-folded at ambient temperature $\left(\chi_{\mathrm{F}}\right.$ of $\left.87 \pm 1 \%\right)$, the most rigid mimics $\mathbf{1 b}$ and $\mathbf{1 c}$ were also characterized by relatively high levels of folding $\chi_{\mathrm{F}}$ of $58 \pm 1 \%$ and $53 \pm 3 \%$ respectively.

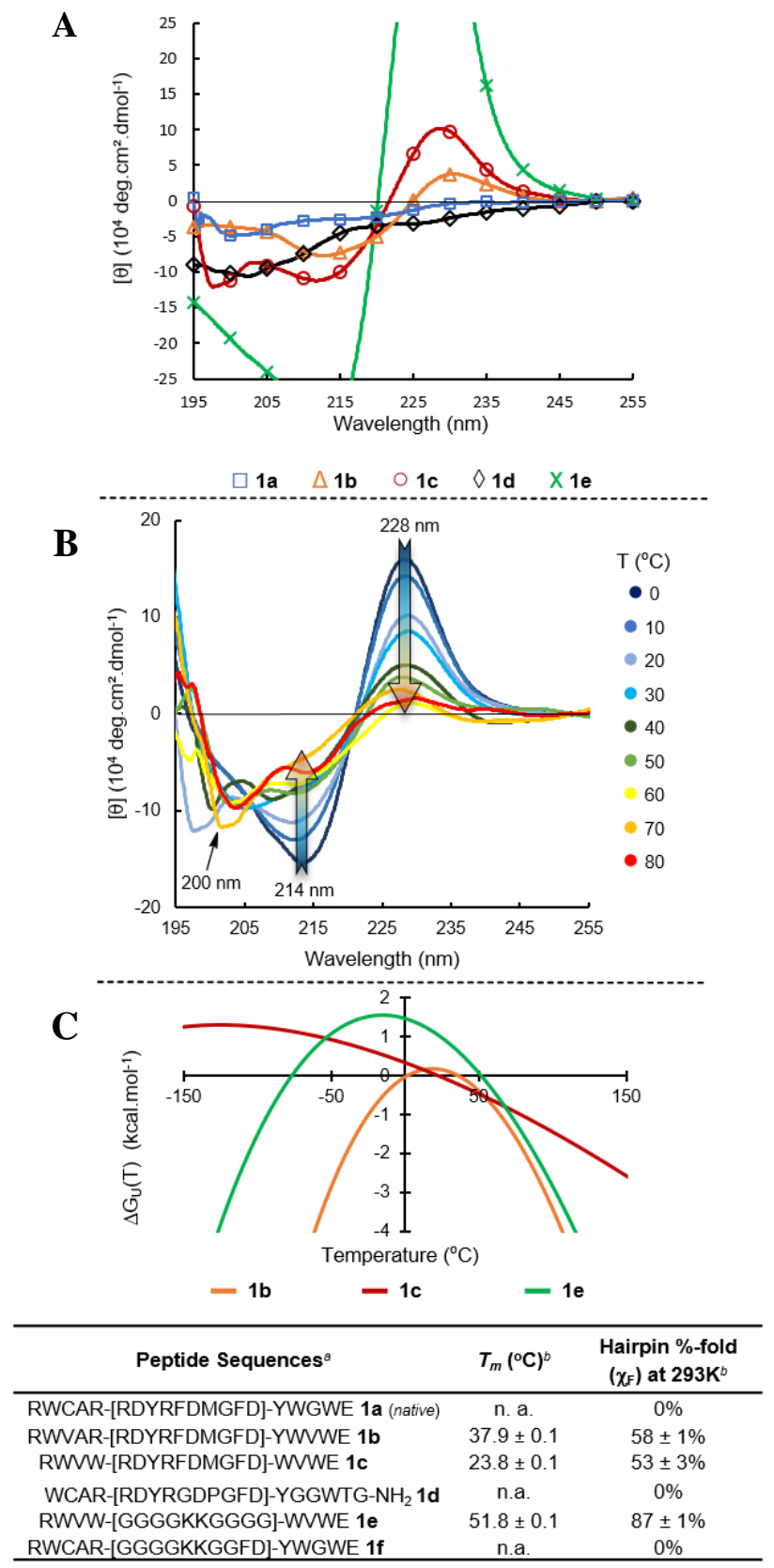

${ }^{a}$ Loop sequences in brackets. ${ }^{b}$ Determined from the best-fitted CD melting curves to the Gibbs-Helmholtz equation for a two-state model.

Figure 5. Comparison of $\beta$-hairpin folding and denaturation properties. (A) Overlay of CD spectra for 1a-e recorded in a phosphate buffer (15 $\mathrm{mM}, \mathrm{pH} 6.5$ ) at $20{ }^{\circ} \mathrm{C}$. (B) Example of temperature dependence on the circular dichroism spectra of hairpin 1c (W/W exciton maxima at $\sim 228$ $\mathrm{nm}$ ) used to plot the melt curve of denaturation. (C) Thermal stability curves showing the free energy of unfolding for hairpins $\mathbf{1 b}, \mathbf{1 c}$ and $\mathbf{1 e}$ as a function of temperature and summary of thermodynamic data.

To confirm the secondary structures suggested by our CD study, a careful structural assignment and conformational analysis was secured by a combination of ${ }^{1} \mathrm{H}$, TOCSY, NOESY and HSQC NMR analyses. The solution structures of hairpins 1b,c and 1e were confirmed based on the large chemical shifts dis- 
persion of $\mathrm{H}_{\mathrm{N}}$ and $\mathrm{H}_{\alpha}$ signals typical of foldamers, and the significant positive and negative chemical shift deviations (CSDs) observed in both strap and loop regions respectively (see Supplementary Figure S10). ${ }^{37}$ The hairpin folds were supported by several strong long-range NOESY correlations between backbone protons of the RW(VW) $\bullet$ (WV)WE $\beta$-strap motif. Together, the NMR data conclusively established that $\beta$-hairpin $\mathbf{1 b}, \mathbf{c}$ and $\mathbf{1 e}$ are folded. On the other end, the NMR study of $\mathbf{1 d}$ and 1f strongly suggest that these peptides are misfolded or exist as unfolded random coils in solution.

The robust loop-display system developed by our group enabled the presentation of the pembrolizumab sequence within several hairpin scaffolds. Hence, we used the display system to evaluate the inhibitory activity of these hairpins in a PD-1:PDL1 binding assay (Figure 6). As positive controls, two commercially available PD-L1 inhibitors (BMS202 and BMS1166), and pembrolizumab (PD-1 blocker) were tested in this assay (see Supplementary Figure S11). The PD-1:PD-L1 inhibition results obtained for the BMS small molecules were consistent with previous reports, with half-maximal inhibitory concentration $\mathrm{IC}_{50}$ values of $0.2 \pm 0.3 \mu \mathrm{M}$ and $0.3 \pm 0.6 \mu \mathrm{M}$ respectively, and a potency of $1.0 \pm 0.3 \mathrm{nM}$ for pembrolizumab. ${ }^{37}$ Not surprisingly, hairpin 1e and its unfolded analog $\mathbf{1 f}$ presenting a polyglycine loop revealed no meaningful blocking activity in the assay $\left(\mathrm{IC}_{50}\right.$ of $3.3 \pm 0.6$ and $1.1 \pm 0.2 \mu \mathrm{M}$ respectively). This result suggests that neither the $\beta$-strap, nor the polyglicinyl loop achieved any significant binding to PD-1. Similarly, construct 1d which was predicted by NMR and CD spectroscopy to have no secondary structure exhibited no measurable activity. Similarly, the native sequence of 1a demonstrated no compelling activity ( IC $_{50}$ of 2.8 $\pm 1.0 \mu \mathrm{M})$ as could be expected from an unfolded construct. Despite its well-folded behavior, $\beta$-hairpin $\mathbf{1 b}$ had a surprisingly modest inhibitory activity in the PD-1:PD-L1 blockade assay $\left(\mathrm{IC}_{50}=1.0 \pm 0.2 \mu \mathrm{M}\right)$. In contrast, hairpin 1c with a more constrained loop flanked by the stabilizing strap motif demonstrated the most potency in a dose dependent manner. Hairpin $R W V W$-[RDYRFDMGFD]- $W V W E$ 1c was shown to achieve a significant blockade of PD-1:PD-L1 interface with an $\mathrm{IC}_{50}$ of $0.6 \pm 0.2 \mu \mathrm{M}$. This result confirms that the loop of the $\beta$-hairpin foldamer has a significant pharmacological activity, and validates the design of pembrolizumab CDR-H3 mimics for the inhibition of PD-1:PD-L1 binding.

\section{Conclusion}

In summary, a comprehensive reinterpretation of the PD1:pembrolizumab interface was carried out from three different co-crystal X-ray structures (Supplementary Tables S2-S4). Three distinct PD-1 epitopes were mapped capturing a detailed blueprint of binding contact interactions with the corresponding paratopes of pembrolizumab for the first time. The remarkable flexibility of both ${ }_{\mathrm{PD}-1} \mathrm{FG}$ and ${ }_{\mathrm{PD}-1} \mathrm{C}^{\prime} \mathrm{D}$ ( $\Omega$-type) loops plays a crucial role in the conformational pre-equilibrium of PD-1 and its binding to pembrolizumab through a dynamic induced-fit mechanism. Morphing and MD simulations further demonstrated that these two distal epitopes possess enough plasticity to bend both edges of the receptor and form a deep binding groove (C'D/FG loops) latching on the pembrolizumab H3 loop (see Supplementary Videos S2-S3). The inherent conformational motion of PD-1 presented herein is consistent with the elasticity reported recently towards PD-L1 $1^{32,57}$ and PD-L2 ${ }^{33}$, and contributes to a better understanding of the overall conformational discrimination mechanism of this receptor. ${ }^{52}$ Central to this binding is the rigid H3 loop $\left[\mathrm{Arg}^{\mathrm{H} 99}-\mathrm{Asp}^{\mathrm{H} 108}\right.$ ] which was shown to create an important number of contacts with residues pertaining to all three epitopes of PD-1, across a large binding interface (38\% of the total BSA). Guided by crystallographic data analysis, we evaluated a rational structure-based approach to derive $\beta$-hairpin mimics of the pembrolizumab $\mathrm{H} 3$ loop as PD-1 inhibitors. Several peptide constructs stabilized by

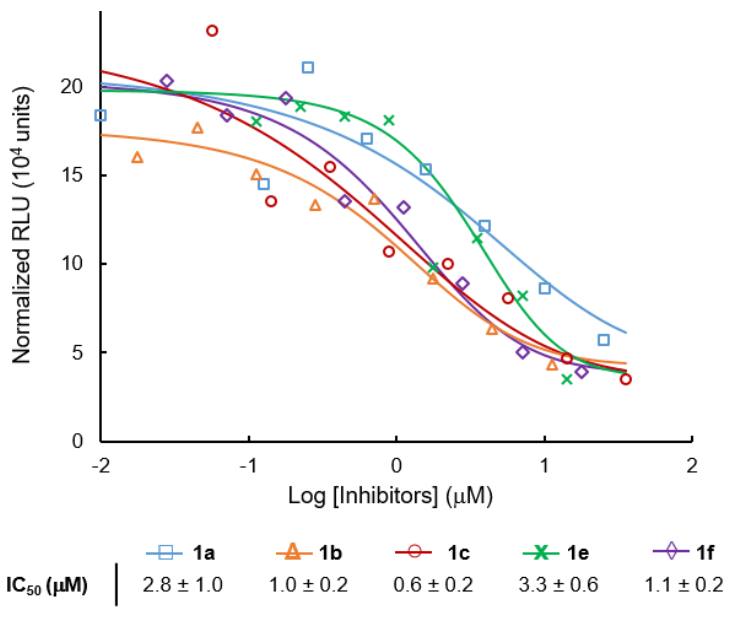

Figure 6. Structure-activity relationship of peptide inhibitors 1a-f of the PD-1/PD-L1 interface. Overlay of dose-response curves for peptide 1a-f obtained by ELISA using varying concentrations of each inhibitor to derive the $\mathrm{IC}_{50}$ values.

a RWV(W/A) $\cdots($ W)VWE $\beta$-strap were shown by $\mathrm{CD}$ and NMR spectroscopy to exist in a $\beta$-hairpin folded conformation. From the initial series of folded $\beta$-hairpins, we identified one promising "hit", $\beta$-hairpin 1c, to inhibit PD-1:PD-L1 interaction with an $\mathrm{IC}_{50}$ of $0.6 \pm 0.2 \mu \mathrm{M}$. The activity and toxicity associated with such $\beta$-hairpin peptides will need to be confirmed on $\mathrm{T}$ cells to further develop promising PD-1 inhibitor candidates. To our knowledge, hairpin 1c is the first non-antibody molecule inhibitor of PD-1 reported to date. This study therefore establishes a proof-of-concept that mimicking the pembrolizumab H3 loop is an attractive approach to generate novel and desirable medium-sized peptide-based PD-1 inhibitors.

\section{METHODS}

X-Ray Crystal Structures Analysis and Interpretation. All co-crystal structures described in this paper were obtained from https://www.rcsb.org/. The interpretation of contact interactions was assisted by LigPlot,$+{ }^{58}$ and the molecular visualization with PyMOL. ${ }^{59}$ Reported PDB codes of pembrolizumabFab complexed with human Programmed Cell-Death protein 1 (PD-1) structures are 5JXE, 5B8C and 5GGS. Those co-crystal structures are formed by di-, tetra- and dimeric assemblies, respectively. In each crystal, the monomer displaying the largest number of contact interactions was selected for analysis, and supplemental interactions found inside other monomers were also included if the residues were not found to be conformational outliers. The summary of polar contacts and hydrophobic interactions are reported in Supplementary Tables S2 and S3 respectively. A maximum cut-off of $2.70 \AA$ for hydrogen bonds: $\mathrm{H}_{\text {ydrogen }}-\mathrm{A}_{\text {cceptor }}$ distance, $4.0 \AA$ for salt bridges: $\mathrm{D}_{\text {onor }}-\mathrm{A}_{\text {cceptor }}$ distance, and a range of 2.90 -3.90 $\AA$ for hydrophobic contacts distances were implemented for the automatized analysis in Ligplot+. 
Loop Modeling and Morphing Simulation. Due to the important plasticity of the C'D loop segment in the apo-PD-1 crystal structure (PDB: 3RRQ), a remodeling and a minimum energy calculation of this loop was necessary to determine a model of stable unbounded conformation. To this aim, the insertion of residue E61 was required prior to minimization. A portion of the C'D loop of PD- $1\left[\mathrm{Pro}^{83}-\mathrm{Arg}^{94}\right]$, including sequence $\mathrm{D}^{85} \mathrm{RSQPGQD}{ }^{92}$ was remodeled to obtain a set of 500 low-energy conformers generated using the loop modeling protocol DOPE-HR from the MODELLER add-on ${ }^{60}$ in the UCSF Chimera Software ${ }^{43}$ (Supplementary Figure S4A). The lowestenergy loop conformer was selected using the zDOPE score related to free energy (zDOPE -1.93 au) which enabled us to obtain an initial wild-type PD-1 model with a reconstituted C'D loop (PDB: 3RRQ*). Secondly, the missing E61 residue was added with Interactive ROSETTA (KIC protocol > SES loop modeling, De Novo) to generate the complete model of the wildtype apo-PD-1(PDB: 3RRQ**) bearing the reconstituted C'D and BC loops shown in Supplementary Figure S4B. In brief, a morphing simulation was achieved to extrapolate the plausible trajectory of PD-1 upon binding by using the remodeled apoPD-1 (PDB: 3RRQ**) as initial state, and a bounded PD-1 structure extracted from the co-crystal with pembrolizumab (PDB: 5GGS) as final state. The new PD-1 model (PDB: $3 \mathrm{RRQ}^{* *}$ ) along with the apo-pembrolizumab (PDB: 3DK3) were overlaid with the reported PD-1:pembrolizumab-Fab Xray complex (PDB: 5GGS) (RMSD $=0.41 \AA$ and $0.74 \AA$ respectively, Supplementary Figure S5, STEP 1). Interpolated trajectories were calculated on each individual chain/object (pembrolizumab heavy-chain, light-chain and PD-1 separately) to obtain a conformational simulation from the apo-forms to the bounded states through a motion comprising 60 states (60 refinement cycles, STEP 2). The 3 objects were reassembled together to obtain the full-length simulation with the motion of both pembrolizumab and PD-1 and water molecules from the 5 GGS crystal were added to the final file to maintain the hydrogen bond and salt bridge contacts involving water (STEP 3). Dynamic polar interactions between the two proteins are displayed in the final state to generate the Supplementary Video S3.

Loop Dynamics Validation. To better understand the plasticity of the PD-1 receptor, both structures apo-PD-1 remodeled (PDB: 3RRQ**) and the PD-1 excised from PDBs 5GGS and 5B8C have been tested for loop flexibility using ToeLoop. ${ }^{44,45}$ Results from these MD simulations were exploited to estimate the difference of motions between loops (Supplementary Figure S6).

Peptide Synthesis. Peptides were prepared by Fmoc solidphase peptide synthesis (SPPS) on a Protein Technologies PS3 peptide synthesizer. A detailed description of the peptide synthesis, cleavage and purity analysis procedures are given under Supporting Information: Peptide Synthesis, Purification, and Structural Characterization.

Nuclear Magnetic Resonance Data Collection. NMR samples were prepared by dissolving the freeze-dried peptide $(\sim 1$ $2 \mathrm{mg})$ in a mixture of phosphate buffer $(50 \mathrm{mM}, \mathrm{pH} 6.5)$ and $\mathrm{D}_{2} \mathrm{O}(9: 1, \mathrm{v} / \mathrm{v})$ for 1d-f, a mixture of phosphate buffer $(50 \mathrm{mM}$, $\mathrm{pH}$ 6.5) and DMSO- $d_{6}(7: 3, \mathrm{v} / \mathrm{v})$ for $1 \mathrm{c}$, and a mixture of water and DMSO- $d_{6}(8: 2, \mathrm{v} / \mathrm{v})$ for $\mathbf{1 b}$, using 2,2-dimethyl-2-silapentane-5-sulfonate (DSS) as internal standard for chemical shifts $(0 \mathrm{ppm})$. Samples were prepared in a range of 3-10 $\mathrm{mM}$ of pep- tide for ${ }^{1} \mathrm{H}$ NMR, TOCSY (mixing time $80 \mathrm{~ms}$ ), NOESY (mixing time $200 \mathrm{~ms}$ ) and ${ }^{1} \mathrm{H}^{-13} \mathrm{C}$ HSQC experiments, and in a range of $10-20 \mathrm{mM}$ to record ${ }^{1} \mathrm{H}_{-}{ }^{15} \mathrm{~N}$ HSQC and HMBC spectra. A PRESAT experiment was used to suppress the $\mathrm{H}_{2} \mathrm{O}$ solvent signal, in order to record the initial ${ }^{1} \mathrm{H}$ NMR. All spectra were recorded at $291 \mathrm{~K}\left(18{ }^{\circ} \mathrm{C}\right)$ on a Varian Mercury500 (500 MHz) spectrometer and processed using the Vnmrj 4.2 software. Signals assignments were obtained on the basis of a set of ${ }^{1} \mathrm{H}$ and TOCSY spectra (for intra-residue connectivities), NOESY spectra (for vicinal and interstrand backbone connectivities), and HSQC spectra (for $\mathrm{H}_{\alpha}$ to $\mathrm{C}_{\alpha}$ connectivities and $\mathrm{W}$ sidechains assignments).

Far-UV Circular Dichroism (CD) Spectroscopy. Peptide solutions were prepared at $20-100 \mu \mathrm{M}$ concentration range in phosphate buffer (15 mM, pH 6.5), with addition of $\mathrm{MeOH}$ (up to $50 \% \mathrm{v} / \mathrm{v}$ ) if required to increase solubility. Each sample concentration was determined accurately by measuring the solution UV-absorbance using a JASCO V-670 spectrophotometer based on the combined molar absorptivity of Trp and Tyr residues at $280 \mathrm{~nm}\left(\varepsilon_{280}=5580 \mathrm{M}^{-1} \cdot \mathrm{cm}^{-1}\right.$ per Trp, $\varepsilon_{280}=1280 \mathrm{M}$ ${ }^{1} . \mathrm{cm}^{-1}$ per Tyr). CD spectra were recorded on a JASCO J-810 Spectropolarimeter with a temperature controller module JASCO PFD-425S. In brief, raw CD data were recorded in mdeg from $185 \mathrm{~nm}$ to $270 \mathrm{~nm}$ every $0.1 \mathrm{~nm}$, and CD spectra of the blank solutions were subtracted for baseline correction. Spectra were smoothed, the baseline was set to 0 between 260 $\mathrm{nm}$ to $270 \mathrm{~nm}$, and the ellipticity scale was converted into molar ellipticity (deg. $\mathrm{cm}^{2} . \mathrm{dmol}^{-1}$ ) using SpectraGryph 1.2. ${ }^{61}$ To obtain the melting curves representing the peptides unfolding transitions, variable-temperature $\mathrm{CD}$ experiment were required. The temperature ramp was controlled automatically from 5 to $95{ }^{\circ} \mathrm{C}$ with a ramp speed of $0.75{ }^{\circ} \mathrm{C} / \mathrm{min}$. Intensity values at $228 \mathrm{~nm}$ were recorded by the instrument every $0.1{ }^{\circ} \mathrm{C}$ to obtain raw melting curves. Likewise, intensities for the corresponding blank solutions were recorded and subtracted to afford the experimental melting curves' output. These melting curves were reported in molar ellipticity $\left(\mathrm{deg} . \mathrm{cm}^{2} \cdot \mathrm{dmol}^{-1}\right)$ divided by the number of tryptophan pairs present in the peptide construct $\left([\theta(\mathrm{T})]_{228} / p \operatorname{Trp}\right)$ in order to normalize the spectra. Finally, a protocol has been developed to compute the best-fitted melting curves from the raw data of $[\theta(\mathrm{T})]_{228}$ as function of temperature by using the thermodynamic Gibbs-Helmholtz equation and a nonlinear least square fitting routine programmed in Origin 9.0 (Originlab Corporation, U.S.A.). A detailed description of the $\%$-folding best-fitted curves, and the thermodynamic calculations to obtain the values for $\Delta G^{\mathrm{o}}, T_{m}, \Delta C_{p}{ }^{o}$ are given under Supporting Information: Peptide Synthesis, Purification, and Structural Characterization.

PD-1 ImmunoBlocking Assay. Peptide inhibitors stock solutions were prepared at $\sim 5 \mathrm{mM}$ concentration range in DMSO. Assay kit for screening and profiling inhibitors of the PD-1/PDL1 interaction was purchased from BPS Bioscience (cat \# 72005). Briefly, a 96-well plate was coated with PD-L1 at $2 \mu \mathrm{g} / \mathrm{mL}$ overnight at $4{ }^{\circ} \mathrm{C}$. Plate was then washed and blocked with blocking buffer for $1 \mathrm{~h}$ at room temperature. Inhibitor solutions (0.01-100 $\mu \mathrm{M})$ and blank (DMSO/PBS buffer solution) were added to designated wells. To initiate the reaction, 0.5 $\mathrm{ng} / \mu \mathrm{L}$ of biotin-labeled human PD- 1 was then added into positive control wells and inhibitor solution wells. After $2 \mathrm{~h}$ incubation at room temperature, plate was washed and blocked again for 10 mins. Streptavidin-HRP (1:1000 dilution) was added and incubate for $1 \mathrm{~h}$ at room temperature with slow shaking. Plate 
was washed and block again for 10 mins. ELISA ECL substrate was then added and the resulting chemiluminescence was immediately read in luminometer (FLUOstar OPTIMA, BMG Labtech). Luminescence value of the blank is subtracted from all readings according to manufacturer instruction. The half maximal effective concentrations $\left(\mathrm{IC}_{50}\right)$ values were determined from the fit of experimental normalized luminescence data to the Hill model (Figure 6) with $\mathrm{RLU}_{\max }$ values representing maximal relative luminescence values from the PD-1/PDL1 interaction. Inhibitory curves were overlaid accordingly to a reported procedure. ${ }^{62}$

\section{ASSOCIATED CONTENT}

\section{Supporting Information}

The Supporting Information is available online. Supplementary data to this article summarizing the X-rays analysis, the C'D loop modeling, the morphing protocols, the synthesis and characterization of peptide inhibitors, as well as Supplementary Videos S1-3 of conformational morphing for the binding of PD-1 with PD-L1 and pembrolizumab, as well as and the PDB file for the reconstituted apo PD-1 ectodomain (3RRQ**) are available online.

\section{AUTHOR INFORMATION}

\section{Corresponding Author}

* Correspondence should be addressed to S.P.R. Email: sroche2@fau.edu

\section{Author Contributions}

This project was conceived by S.P.R. and N. S. Epitopes/paratopes mapping was achieved by S.P.R. and M.Z., and the loop modeling, Molecular dynamics and morphing simulation were carried out by M.Z.. A.D.R. and G.Z. synthesized all peptide inhibitors and analyzed the structural and folding data. M.W. and N.S. provided the biological evaluation. S.P.R. wrote the manuscript with the contribution of M.Z. and A.D.R. All authors have given approval to the final version of the manuscript.

\section{ACKNOWLEDGMENT}

We are very grateful for the financial support from the US National Institutes of Health (NIGMS Grant: R15GM116025 to M.Z., and Grant: R21GM132754 to S.P.R.). We thank Dr. M. Cudic and Dr. D. Du at Florida Atlantic University for the use of their peptide synthesizer. The authors also thank Dr. Giovanni GonzalezGutierrez from the Macromolecular Crystallography Facility at Indiana University Bloomington for the stimulating discussions and sharing his expertise on the reinterpretation of the various crystal structures studied herein and Dr. G. Theodore from Theogen Corp. for manuscript proofreading and editing.

\section{DECLARATION of INTERESTS}

The authors declare no competing interests.

\section{REFERENCES}

(1) Pardoll, D. M. The blockade of immune checkpoints in cancer immunotherapy. Nat. Rev. Cancer 2012, 12, 252-264.

(2) Lee, L.; Gupta, M.; Sahasranaman, S., Immune Checkpoint inhibitors: An introduction to the next-generation cancer immunotherapy. $J$. Clin. Pharmacol. 2016, 56, 157-169.

(3) Boussiotis, V. A. Molecular and Biochemical Aspects of the PD-1 Checkpoint Pathway. N. Engl. J. Med. 2016, 375, 1767-1778.

(4) Goodman, A.; Patel, S. P.; Kurzrock, R. PD-1-PD-L1 immunecheckpoint blockade in B-cell lymphomas. Nat. Rev. Clin. Oncol. 2017 $14,203-220$.
(5) De Sousa Linhares, A.; Leitner, J.; Grabmeier-Pfistershammer, K.; Steinberger, P., Not All Immune Checkpoints Are Created Equal. Front. Immunol. 2018, 9, 1909.

(6) Chen, D. S.; Irving, B. A.; Hodi, F. S. Molecular Pathways: NextGeneration Immunotherapy-Inhibiting Programmed Death-Ligand 1 and Programmed Death-1. Clin. Cancer Res. 2012, 18, 6580-6587.

(7) Luke, J. J.; Ott, P. A. PD-1 pathway inhibitors: the next generation of immunotherapy for advanced melanoma. Oncotarget 2015, 6, 34793492.

(8) Scapin, G., Yang, X., Prosise, W. W., McCoy, M., Reichert, P., Johnston, J. M., Kashi, R. S., Strickland, C. Structure of full-length human anti-PD1 therapeutic IgG4 antibody pembrolizumab. Nat. Struct. Mol. Biol. 2015, 22, 953-958.

(9) Tang, J., Yu, J. X., Hubbard-Lucey, V. M., Neftelinov, S. T., Hodge, J. P., Lin, Y. The clinical trial landscape for PD1/PDL1 immune checkpoint inhibitors. Nat. Rev. Drug Discov. 2018, 17, 854855 .

(10) Wang, X.; Teng, F.; Kong, L.; Yu, J., PD-L1 expression in human cancers and its association with clinical outcomes. OncoTargets Ther. 2016, 9, 5023-5039.

(11) O'Donnell, J. S.; Long, G. V.; Scolyer, R. A.; Teng, M. W. L.; Smyth, M. J., Resistance to PD1/PDL1 checkpoint inhibition. Cancer Treat. Rev. 2017, 52, 71-81.

(12) Nowicki, T. S.; Hu-Lieskovan, S.; Ribas, A., Mechanisms of Resistance to PD-1 and PD-L1 Blockade. Cancer J. 2018, 24, 47-53.

(13) Narváez, J.; Juarez-López, P.; Lluch, J.; Narváez, J. A.; Palmero, R.; García del Muro, X.; Nolla, J. M.; Domingo-Domenech, E., Rheumatic immune-related adverse events in patients on anti-PD-1 inhibitors: Fasciitis with myositis syndrome as a new complication of immunotherapy. Autoimmun. Rev. 2018, 17, 1040-1045.

(14) Wang, Y.; Zhou, S.; Yang, F.; Qi, X.; Wang, X.; Guan, X.; Shen, C.; Duma, N.; Vera Aguilera, J.; Chintakuntlawar, A.; Price, K. A.; Molina, J. R.; Pagliaro, L. C.; Halfdanarson, T. R.; Grothey, A.; Markovic, S. N.; Nowakowski, G. S.; Ansell, S. M.; Wang, M. L., Treatment-Related Adverse Events of PD-1 and PD-L1 Inhibitors in Clinical Trials: A Systematic Review and Meta-analysis. JAMA Oncol. 2019, 5, 1008-1019.

(15) Sharpe, A. H., Pauken, K. E. The diverse functions of the PD1 inhibitory pathway. Nat. Rev. Immunol. 2018, 18, 153-167.

(16) Lepir, T.; Zaghouani, M.; Roche, S. P.; Li, Y.-Y.; Suarez, M.; Irias, M. J.; Savaraj, N., Nivolumab to pembrolizumab switch induced a durable melanoma response: A case report. Medicine 2019, 98, e13804.

(17) Na, Z., Yeo, S. P., Bharath, S. R., Bowler, M. W., Balıkçı, E., Wang, C.-I., Song, H. Structural basis for blocking PD-1-mediated immune suppression by therapeutic antibody pembrolizumab. Cell Res. 2017, 27, 147-150.

(18) M. M. Miller, C. Mapelli, M. P. Allen, M. S. Bowshe, K. M. Boy, E. P. Gillis, D. R. Langley, E. Mull, M. A. Poirier, N. Sanghvi, L.-Q. Sun, D. J. Tenney, K.-S. Yeung, J. Zhu, P. C. Reid, P. M. Scola, Macrocyclic inhibitors of the pd-1/pd-11 and cd80(b7-1)/pd-11 protein/protein interactions. (Bristol-Myers Squibb Company), U.S. patent 20140294898 A1, Oct 02, 2014

(19) Skalniak, L.; Zak, K. M.; Guzik, K.; Magiera, K.; Musielak, B.; Pachota, M.; Szelazek, B.; Kocik, J.; Grudnik, P.; Tomala, M.; Krzanik, S.; Pyrc, K.; Dömling, A.; Dubin, G.; Holak, T. A. Small-molecule inhibitors of PD-1/PD-L1 immune checkpoint alleviate the PD-L1induced exhaustion of T-cells. Oncotarget 2017, 8, 72167.

(20) Katarzyna, M. M.; Lukasz, S.; M., Z. K.; Bogdan, M.; Ewa, R. S.; Łukasz, B.; Justyna, K.; Przemyslaw, G.; Dominik, S.; Tryfon, Z. T.; Shabnam, S.; Alexander, D.; Grzegorz, D.; A., H. T. Bioactive Macrocyclic Inhibitors of the PD-1/PD-L1 Immune Checkpoint. Angew. Chem. Int. Ed. 2017, 56, 13732.

(21) Guzik, K.; Zak, K. M.; Grudnik, P.; Magiera, K.; Musielak, B.; Törner, R.; Skalniak, L.; Dömling, A.; Dubin, G.; Holak, T. A. SmallMolecule Inhibitors of the Programmed Cell Death-1/Programmed 
Death-Ligand 1 (PD-1/PD-L1) Interaction via Transiently Induced Protein States and Dimerization of PD-L1. J. Med. Chem. 2017, 60, 5857.

(22) For a recent overview small-molecule inhibitors of PD-L1, see: Ganesan, A.; Ahmed, M.; Okoye, I.; Arutyunova, E.; Babu, D.; Turnbull, W. L.; Kundu, J. K.; Shields, J.; Agopsowicz, K. C.; Xu, L.; Tabana, Y.; Srivastava, N.; Zhang, G.; Moon, T. C.; Belovodskiy, A.; Hena, M.; Kandadai, A. S.; Hosseini, S. N.; Hitt, M.; Walker, J.; Smylie, M.; West, F. G.; Siraki, A. G.; Lemieux, M. J.; Elahi, S.; Nieman, J. A.; Tyrrell, D. L.; Houghton, M.; Barakat, K., Comprehensive in vitro characterization of PD-L1 small molecule inhibitors. Sci. Rep. 2019, 9, 12392. See also references cited herein.

(23) Richaud, D. A.; Zhao, G.; Hobloss, S.; Roche, S. P., Folding in Place: Design of $\beta$-Strap Motifs to Stabilize the Folding of Hairpins with Long Loops. ChemRxiv (preprint) 2020, https://doi.org/10.26434/chemrxiv.12912452.v2.

(24) Goh, C.-S., Milburn, D., Gerstein, M. Conformational changes associated with protein-protein interactions. Curr. Opin. Struc. Biol. 2004, 14, 104-109.

(25) Dobbins, S. E.; Lesk, V. I.; Sternberg, M. J. E. Insights into protein flexibility: The relationship between normal modes and conformational change upon protein-protein docking. Proc. Nat. Acad. Sci. USA 2008, 105, 10390-10395.

(26) (a) Lexa, K. W.; Carlson, H. A. Full Protein Flexibility Is Essential for Proper Hot-Spot Mapping. J. Am. Chem. Soc. 2011, 133, 200-202.

(27) For a recent review, see: Dishman, A. F.; Volkman, B. F. Unfolding the Mysteries of Protein Metamorphosis. ACS Chem. Biol. 2018 $13,1438-1446$

(28) Pascolutti, R., Sun, X., Kao, J., Maute, R. L., Ring, A. M., Bowman, G. R., Kruse, A. C. Structure and Dynamics of PD-L1 and an Ultra-High-Affinity PD-1 Receptor Mutant. Structure 2016, 24, 17191728.

(29) Cheng, X., Veverka, V., Radhakrishnan, A., Waters, L. C. Muskett, F. W., Morgan, S. H., Huo, J., Yu, C., Evans, E. J., Leslie, A. J., Griffiths, M., Stubberfield, C., Griffin, R., Henry, A. J., Jansson, A., Ladbury, J. E., Ikemizu, S., Carr, M. D., Davis, S. J. Structure and Interactions of the Human Programmed Cell Death 1 Receptor. J. Biol. Chem. 2013, 288, 11771-11785.

(30) Kumar, S.; Ma, B.; Tsai, C.-J.; Sinha, N.; Nussinov, R. Folding and binding cascades: Dynamic landscapes and population shifts. Protein Sci. 2000, 9, 10-19.

(31) Grünberg, R.; Nilges, M.; Leckner, J. Flexibility and Conformational Entropy in Protein-Protein Binding. Structure 2006, 14, 683693.

(32) Liu, W.; Huang, B.; Kuang, Y.; Liu, G., Molecular dynamics simulations elucidate conformational selection and induced fit mechanisms in the binding of PD-1 and PD-L1. Mol. BioSyst. 2017, 13, 892-900.

(33) Tang, S.; Kim, P. S., A high-affinity human PD-1/PD-L2 complex informs avenues for small-molecule immune checkpoint drug discovery. Proc. Nat. Acad. Sci. USA 2019, 116, 24500-24506.

(34) Chen, D.; Tan, S.; Zhang, H.; Wang, H.; He, W.; Shi, R.; Tong, Z.; Zhu, J.; Cheng, H.; Gao, S.; Chai, Y.; Qi, J.; Xiao, M.; Yan, J.; Gao, G. F., The FG Loop of PD-1 Serves as a "Hotspot" for Therapeutic Monoclonal Antibodies in Tumor Immune Checkpoint Therapy. iScience 2019, 14, 113-124.

(35) Horita, S., Nomura, Y., Sato, Y., Shimamura, T., Iwata, S., Nomura, N. High-resolution crystal structure of the therapeutic antibody pembrolizumab bound to the human PD-1. Sci. Rep. 2016, 6, 35297-35304.

(36) Lee, J. Y., Lee, H. T., Shin, W., Chae, J., Choi, J., Kim, S. H., Lim, H., Won Heo, T., Park, K. Y., Lee, Y. J., Ryu, S. E., Son, J. Y., Lee, J. U., Heo, Y.-S. Structural basis of checkpoint blockade by monoclonal antibodies in cancer immunotherapy. Nat. Commun. 2016, 7, 13354 13363.

(37) See Supporting Information for complete experimental details.
(38) Lin, D. Y.-W., Tanaka, Y., Iwasaki, M., Gittis, A. G., Su, H.-P. Mikami, B., Okazaki, T., Honjo, T., Minato, N., Garboczi, D. N. The PD-1/PD-L1 complex resembles the antigen-binding Fv domains of antibodies and T cell receptors. Proc. Nat. Acad. Sci. USA 2008, 105, 3011-3016.

(39) Zak, K. M., Kitel, R., Przetocka, S., Golik, P., Guzik, K., Musielak, B., Dömling, A., Dubin, G., Holak, T. A. Structure of the Complex of Human Programmed Death 1, PD-1, and Its Ligand PDL1. Structure 2015, 23, 2341-2348.

(40) Fessas, P., Lee, H., Ikemizu, S., Janowitz, T. A molecular and preclinical comparison of the PD-1-targeted T-cell checkpoint inhibitors nivolumab and pembrolizumab. Semin. Oncol. 2017, 44, 136-140.

(41) Wells, J. A., McClendon, C. L. Reaching for high-hanging fruit in drug discovery at protein-protein interfaces. Nature 2007, 450, 10011009 .

(42) Shehu, A., Kavraki, L.E. Modeling Structures and Motions of Loops in Protein Molecules. Entropy 2012, 14, 252-290.

(43) Pettersen, E. F., Goddard, T. D., Huang, C. C., Couch, G. S., Greenblatt, D. M., Meng, E. C., Ferrin, T. E. UCSF Chimera-A visualization system for exploratory research and analysis. J. Comput. Chem. 2004, 25, 1605-1612.

(44) Gu, Y., Li, D.-W., Brüschweiler, R. Decoding the Mobility and Time Scales of Protein Loops. J. Chem. Theory Comput. 2015, 11, 1308-1314.

(45) Gu, Y.; Li, D.-W.; Brüschweiler, R. Statistical database analysis of the role of loop dynamics for protein-protein complex formation and allostery. Bioinformatics 2017, 33, 1814-1819.

(46) Leszczynski, J., Rose, G. Loops in globular proteins: a novel category of secondary structure. Science 1986, 234, 849-855.

(47) Fetrow, J. S. Omega loops: nonregular secondary structures significant in protein function and stability. FASEB J. 1995, 9, 708-717.

(48) Lázár-Molnár, E., Yan, Q., Cao, E., Ramagopal, U., Nathenson, S. G., Almo, S. C. Crystal structure of the complex between programmed death-1 (PD-1) and its ligand PD-L2. Proc. Nat. Acad. Sci. USA 2008, 105, 10483-10488.

(49) Yu, J.; Zhou, Y.; Tanaka, I.; Yao, M., Roll: a new algorithm for the detection of protein pockets and cavities with a rolling probe sphere. Bioinformatics 2009, 26, 46-52.

(50) Gavenonis, J., Sheneman, B. A., Siegert, T. R., Eshelman, M. R., Kritzer, J. A. Comprehensive analysis of loops at protein-protein interfaces for macrocycle design. Nat. Chem. Biol. 2014, 10, 716-722.

(51) Papaleo, E.; Saladino, G.; Lambrughi, M.; Lindorff-Larsen, K.; Gervasio, F. L.; Nussinov, R., The Role of Protein Loops and Linkers in Conformational Dynamics and Allostery. Chem. Rev. 2016, 116 , 6391-6423.

(52) Amaral, M., Kokh, D. B., Bomke, J., Wegener, A., Buchstaller, H. P., Eggenweiler, H. M., Matias, P.; Sirrenberg, C., Wade, R. C., Frech, M. Protein conformational flexibility modulates kinetics and thermodynamics of drug binding. Nat. Commun. 2017, 8, 2276-2289.

(53) Anderson, J. M.; Kier, B. L.; Shcherbakov, A. A.; Andersen, N. $\mathrm{H}$. An improved capping unit for stabilizing the ends of associated $\beta$ strands. FEBS Lett. 2014, 588, 4749-4753.

(54) Anderson, J. M.; Shcherbakov, A. A.; Kier, B. L.; Kellock, J.; Shu, I.; Byrne, A. L.; Eidenschink, L. A.; Andersen, N. H. Optimization of a $\beta$-sheet-cap for long loop closure. Biopolymers 2017, 107, e22995.

(55) Anderson, J. M.; Kier, B. L.; Jurban, B.; Byrne, A.; Shu, I.; Eidenschink, L. A.; Shcherbakov, A. A.; Hudson, M.; Fesinmeyer, R. M.; Andersen, N. H. Aryl-aryl interactions in designed peptide folds: Spectroscopic characteristics and optimal placement for structure stabilization. Biopolymers 2016, 105, 337-356.

(56) Greenfield, N. J. Using circular dichroism collected as a function of temperature to determine the thermodynamics of protein unfolding and binding interactions. Nat. Protoc. 2006, 1, 2527-2535. 
(57) During the preparation of this manuscript, a novel computational study on PD-1 flexibility was published: Mittal, L.; Srivastava, M.; Kumari, A.; Tonk, R. K.; Awasthi, A.; Asthana, S., Interplay among Structural Stability, Plasticity, and Energetics Determined by Conformational Attuning of Flexible Loops in PD-1. J. Chem. Inf. Model. 2021, 61, 358-384.

(58) Laskowski, R. A.; Swindells, M. B. LigPlot+: Multiple LigandProtein Interaction Diagrams for Drug Discovery. J. Chem. Inf. Model. 2011, 51, 2778-2786.
(59) The PyMOL Molecular Graphics System, Version 2.0 Schrödinger, LLC.

(60) Webb, B.; Sali, A. Comparative Protein Structure Modeling Using MODELLER. Curr. Protoc. Bioinform. 2016, 54, 5.6.1-5.6.37.

(61) Menges, F. "Spectragryph - optical spectroscopy software", Version 1.2.13, 2019, http://www.effemm2.de/spectragryph/ (accessed May 11, 2020)

(62) Gadagkar, S. R.; Call, G. B., Computational tools for fitting the Hill equation to dose-response curves. J. Pharmacol. Tox. Met. 2015, $71,68-76$ 